\title{
Poles in the S-matrix of relativistic Chern-Simons matter theories from quantum mechanics
}

\section{Yogesh Dandekar, Mangesh Mandlik and Shiraz Minwalla}

Department of Theoretical Physics, Tata Institute of Fundamental Research, Homi Bhabha Road, Mumbai 400005, India

E-mail: yogesh@theory.tifr.res.in, mangesh@theory.tifr.res.in, minwalla@theory.tifr.res.in

ABSTRACT: An all orders formula for the S-matrix for $2 \rightarrow 2$ scattering in large N ChernSimons theory coupled to a fundamental scalar has recently been conjectured. We find a scaling limit of the theory in which the pole in this S-matrix is near threshold. We argue that the theory must be well described by non-relativistic quantum mechanics in this limit, and determine the relevant Schroedinger equation. We demonstrate that the S-matrix obtained from this Schroedinger equation agrees perfectly with this scaling limit of the relativistic S-matrix; in particular the pole structures match exactly. We view this matching as a nontrivial consistency check of the conjectured field theory S-matrix.

Keywords: Scattering Amplitudes, Chern-Simons Theories, Anyons, 1/N Expansion

ARXIV EPRINT: 1407.1322 


\section{Contents}

1 Introduction, analysis and conclusions 1

A The quantum mechanics of anyons with point like interactions $\quad 6$

$\begin{array}{ll}\text { A.1 Quantum mechanics with a two dimensional delta function } & 7\end{array}$

$\begin{array}{lll}\text { A.1.1 Renormalization of the coupling constant } & 7\end{array}$

A.1.2 Description in terms of modified boundary conditions 8

$\begin{array}{ll}\text { A.2 Derivation of the scattering amplitude } & 9\end{array}$

\section{Introduction, analysis and conclusions}

Pure Chern-Simons theories in three dimensions are topological and have no local degrees of freedom; these theories have been studied in great detail over the last three decades starting with the classic work of Witten [1].

Relativistic Chern-Simons theories minimally coupled to charged matter fields have been studied much less than pure Chern-Simons theories. ${ }^{1}$ These theories are non topological, have local degrees of freedom and are not generically solvable. However it has recently been realized that $\mathrm{U}(N)$ level $\kappa$ Chern-Simons theories coupled to fundamental matter are effectively solvable in the 't Hooft large $N$ limit, $N \rightarrow \infty$ and $\kappa \rightarrow \infty$ with $\frac{N}{\kappa}=\lambda$ held fixed. ${ }^{2}$ Three point correlators and the thermal partition function have been computed for such theories to all orders in the 't Hooft coupling $\lambda$; the results of these computations have motivated the conjecture for non supersymmetric strong weak coupling bosonization type dualities between pairs of these theories, and also for conjectured Vasiliev bulk duals for these theories. ${ }^{3}[10-24]$

The recent paper [9] has initiated the study of the S-matrix in fundamental matter Chern-Simons theories to all orders in the 't Hooft coupling. In particular the authors of [9] have presented a detailed study of $2 \rightarrow 2$ scattering in the most general renormalizable theory of a single fundamental scalar interacting with a $\mathrm{U}\left(N_{B}\right)$ Chern-Simons gauge field

$$
S=\int d^{3} x\left[i \varepsilon^{\mu \nu \rho} \frac{\kappa_{B}}{4 \pi} \operatorname{Tr}\left(A_{\mu} \partial_{\nu} A_{\rho}-\frac{2 i}{3} A_{\mu} A_{\nu} A_{\rho}\right)+D_{\mu} \bar{\phi} D^{\mu} \phi+m_{B}^{2} \bar{\phi} \phi+\frac{1}{2 N_{B}} b_{4}(\bar{\phi} \phi)^{2}\right],
$$

\footnotetext{
${ }^{1}$ Non-relativistic versions of these theories describe interactions of anyonic particles, and have been studied in various guises over the last few decades, starting with the work of Aharonov and Bohm [2] (see e.g. the book [3] for a review and [4-8] for more recent work).

${ }^{2} \kappa$ is the level of the Chern-Simons term in the bulk Lagrangian in the dimensional reduction scheme. It is defined as $\kappa=\operatorname{sgn}(k)(|k|+N)$, where $|k|$ is the level of the WZW theory dual to the pure Chern-Simons theory. Please note that $\kappa$ used in this paper $=k$ used in [9].

${ }^{3}$ In a parallel development there has been an explosion of exact results for protected quantities (indices, partition functions etc.) for supersymmetric Chern-Simons theories, obtained using the methods of supersymmetric localization. As it is possible to construct supersymmetric theories with matter in the fundamental representation, it should be possible - and would be fascinating - to make connections between these as yet distinct streams of work.
} 
to all orders in $\lambda_{B}=\frac{N_{B}}{\kappa_{B}}$.

The theory (1.1) has elementary quanta that transform in either the fundamental or the antifundamental representations of $\mathrm{U}\left(N_{B}\right)$. Following the terminology of [9], we refer to quanta in the fundamental representation as particles, and quanta in the antifundamental representation as antiparticles. The authors of [9] were able to explicitly compute the particle - particle scattering matrix together with the particle - antiparticle scattering matrix in the channel corresponding to adjoint exchange. They also presented the following conjectured formula for the particle - antiparticle S-matrix in the channel corresponding to singlet exchange:

$$
\begin{aligned}
& T_{S}(\sqrt{s}, \theta)=8 \pi i \sqrt{s}\left(1-\cos \left(\pi \lambda_{B}\right)\right) \delta(\theta)+4 i \sqrt{s} \sin \left(\pi \lambda_{B}\right) \operatorname{Pv}\left(\cot \left(\frac{\theta}{2}\right)\right) \\
& +4 \sqrt{s} \sin \left(\pi\left|\lambda_{B}\right|\right)\left(\frac{\left(4 \pi\left|\lambda_{B}\right| \sqrt{s}+\widetilde{b}_{4}\right)+e^{i \pi\left|\lambda_{B}\right|}\left(-4 \pi\left|\lambda_{B}\right| \sqrt{s}+\widetilde{b}_{4}\right)\left(\frac{\frac{1}{2}+\frac{c_{B}}{\sqrt{s}}}{\frac{1}{2}-\frac{c_{B}}{\sqrt{s}}}\right)^{\left|\lambda_{B}\right|}}{\left(4 \pi\left|\lambda_{B}\right| \sqrt{s}+\widetilde{b}_{4}\right)-e^{i \pi\left|\lambda_{B}\right|}\left(-4 \pi\left|\lambda_{B}\right| \sqrt{s}+\widetilde{b}_{4}\right)\left(\frac{\frac{1}{2}+\frac{c_{B}}{\sqrt{s}}}{\frac{1}{2}-\frac{c_{B}}{\sqrt{s}}}\right)^{\left|\lambda_{B}\right|}}\right),
\end{aligned}
$$

where

$$
\begin{aligned}
c_{B} & =\text { pole mass of the single scalar excitation, } \\
\sqrt{s} & =\text { centre of mass energy } \\
\theta & =\text { angle of scattering, } \\
\widetilde{b}_{4} & =2 \pi \lambda_{B}^{2} c_{B}-b_{4} .
\end{aligned}
$$

As explained in [9], the S-matrix (1.2) does not agree with the simple analytic continuation of the particle - particle S-matrix. Instead, the nonsingular part of (1.2) is given by the analytic continuation of the particle - particle S-matrix rescaled by the factor $\frac{\sin \left(\pi \lambda_{B}\right)}{\pi \lambda_{B}}$. In other words the correctness of the conjectured S-matrix (1.2) requires an intriguing modification of the usual text book rules of crossing symmetry in the case of matter Chern-Simons theories. As with any conjecture that challenges accepted wisdom, the formula (1.2) should be subjected to stringent checks. In this note we confront the conjecture of [9] with a nontrivial consistency check and find that it passes the test, as we now describe.

The S-matrix (1.2) has a pole for $\widetilde{b}_{4} \geq \widetilde{b}_{4}^{\text {crit }}=8 \pi c_{B}\left|\lambda_{B}\right|$ indicating the existence of a particle - antiparticle bound state in the singlet channel at these values of parameters. ${ }^{4}$ As $\widetilde{b}_{4}$ approaches $\widetilde{b}_{4}^{\text {crit }}$ from above, the mass of the bound state approaches $2 c_{B}$. In other words, if we set $\widetilde{b}_{4}=\widetilde{b}_{4}^{\text {crit }}+\delta b_{4}$, the binding energy $E_{B}$ is small at small $\delta b_{4}$ (it turns out $\left.E_{B} \sim\left(\delta b_{4}\right)^{1 /\left|\lambda_{B}\right|}\right)^{5}$ and vanishes when $\delta b_{4}=0$.

\footnotetext{
${ }^{4} b_{4}$ is always negative when bound states exist, so it possible that (1.1) is non perturbatively unstable in this range of parameters. While the study of the nonperturbative stability of (1.1) is an interesting question (one that can presumably be settled by the evaluation of the all orders effective action for $\phi$ ), it is irrelevant for the perturbative considerations of this note, and will not be studied in this paper.

${ }^{5}$ More precisely, at lowest nontrivial order in $\delta b_{4}$
}

$$
\frac{E_{B}}{4 c_{B}}=\left(\frac{\delta b_{4}}{16 \pi\left|\lambda_{B}\right| c_{B}}\right)^{\frac{1}{\left|\lambda_{B}\right|}}
$$


Motivated by this observation, in this note we focus on the field theory (1.1) in a sector containing a singlet particle - antiparticle pair in a particular scaling limit we call the 'near threshold limit'. This limit is defined by scaling $\delta b_{4}$ to zero while simultaneously scaling $\sqrt{s}-2 c_{B}$ to zero like $\left(\delta b_{4}\right)^{1 /\left|\lambda_{B}\right|}$. In this limit the particles are non-relativistic and we may set $\sqrt{s}-2 c_{B}=\frac{k^{2}}{c_{B}} .{ }^{6}$ In our scaling limit

$$
\frac{\delta b_{4}}{c_{B}} \rightarrow 0, \quad \frac{k}{c_{B}} \rightarrow 0, \quad \frac{k}{c_{B}}\left(\frac{c_{B}}{\delta b_{4}}\right)^{\frac{1}{2\left|\lambda_{B}\right|}}=\text { fixed } .
$$

Like any non-relativistic limit, our limit focuses attention on a sector of the theory in which kinetic energies of the particle and antiparticle are small compared to rest masses. In this limit our system must admit an effective description in terms of the non-relativistic quantum mechanics of two particles interacting via Chern-Simons gauge boson exchange, plus a contact interaction. We will now describe this quantum mechanical system in more detail, following Amelino-Camelia and Bak [7].

It is well known (see, for instance, $[5,6]$ ) that the entire effect of the Chern-Simons interactions between non-relativistic particles is to implement anyonic statistics for the particles. This happens because the Chern-Simons equation of motion forces each particle to trap a unit of flux; the other particle picks up a phase when circumnavigating this flux. The magnitude of the phase depends on the coupling colour factors: when the colour factors of the two particles (which transform in representations $R_{1}$ and $R_{2}$ respectively) Clebsch-Gordon couple into representation $R_{m}$ it turns out that the magnitude of the phase is given by [5]

$$
\nu_{m}=\frac{c_{2}\left(R_{m}\right)-c_{2}\left(R_{1}\right)-c_{2}\left(R_{2}\right)}{\kappa},
$$

where $c_{2}(R)$ is the quadratic Casimir of the representation $R$.

The effect of this phase is most simply described when we change variables to work with the centre of mass and relative degrees of freedom of the particle - antiparticle system. The centre of mass motion is free, and is ignored in what follows. In terms of relative coordinates, in the gauge singlet sector (i.e. $c_{2}\left(R_{m}\right)=0$ ), the entire effect of the Chern-Simons coupled gauge field is implemented by inserting a point like solenoid of integrated flux $-2 \pi \lambda_{B}$ at the origin of the two dimensional plane. The quantum mechanical description of this system is given by a non-relativistic Schroedinger equation (1.7) below for a particle of effective mass $\frac{c_{B}}{2}$ and of effective $\mathrm{U}(1)$ charge unity, minimally coupled to a $\mathrm{U}(1)$ gauge field corresponding to this point like solenoid (see the section (2.6) of [9] and references therein). In other words, the time independent Schroedinger equation for our system at energy $E=\sqrt{s}-2 c_{B}=\frac{k^{2}}{c_{B}}$ is given by

$$
\begin{aligned}
-D_{i} D^{i} \psi & =k^{2} \psi, \\
D_{i} & =\nabla_{i}+i A_{i}, \\
A_{i} & =\nu \frac{\epsilon_{i j} x^{j}}{x^{2}},
\end{aligned}
$$

\footnotetext{
${ }^{6}$ Note that our definition of the near threshold limit does not constrain $\sqrt{s}-2 c_{B}$ to take a particular sign. This quantity is negative in the study of bound states, and positive in the study of scattering.
} 
where, in the singlet sector, (as in (2.47) of [9])

$$
\nu=-\lambda_{B}
$$

It turns out that the point like interaction between the particle and the antiparticle imposes modified boundary conditions for this effective Schroedinger wave function at origin [7, 25](see the appendix A.1.2 for an intuitive explanation). As explained in [7, 25] there exists a one parameter set of consistent and self-adjoint boundary conditions for the wave function at the origin. These boundary conditions are specified as follows. Let

$$
\psi(\vec{r})=\sum_{m} e^{i m \theta} \psi_{m}(r) .
$$

The functions $\psi_{m}(r)$ for $m \neq 0$ are required, as usual to vanish at $r=0$. For $m=0$, on the other hand, we require that

$$
\psi_{0}(r) \propto\left(r^{\left|\lambda_{B}\right|}+\frac{w R^{2\left|\lambda_{B}\right|}}{r^{\left|\lambda_{B}\right|}}\right),
$$

where $R$ is a reference length scale and $w$ is the self-adjoint extension parameter as introduced in [7].

In other words $\psi_{0}$ is not forced to vanish at the origin but has a component that blows up. We refer to (1.10) as the Amelino-Camelia-Bak boundary conditions.

The modified boundary conditions (1.10) are labeled by the single dimensionful parameter $w R^{2\left|\lambda_{B}\right|}$. It follows from dimensional analysis that the effect of this parameter on any process with characteristic momentum scale $k$ (like the scattering of particles with momentum $k$ ) is proportional to $w(R k)^{2\left|\lambda_{B}\right|}$. As $w(R k)^{2\left|\lambda_{B}\right|} \rightarrow 0$ the boundary conditions above effectively reduce to the 'usual' Aharonov-Bohm boundary conditions; the boundary conditions that force $\psi_{0}$ to vanish at the origin.

In summary, the low energy effective description of the particle - antiparticle system in the near threshold limit is given by the quantum mechanics of a single non-relativistic particle propagating in two dimensions. The wave function of this particle obeys the Schroedinger equation (1.7) and the boundary conditions (1.10). The boundary condition parameter $w R^{2\left|\lambda_{B}\right|}$ in (1.10) is an as yet unknown function of $\delta b_{4}$.

It follows from the discussion above that the S-matrix (1.2) must reduce in the near threshold limit, to the S-matrix computed by solving (1.7) subject to the Amelino-CameliaBak boundary conditions. This expectation is a nontrivial consistency check of the conjecture (1.2), which we now proceed to verify.

The near threshold limit of the S-matrix (1.2) is easily determined. As above we set

$$
\sqrt{s}=2 c_{B}+\frac{k^{2}}{c_{B}} \text {. }
$$

In the limit (1.5), the second line of (1.2) reduces to

$$
8 c_{B}\left|\sin \left(\pi \lambda_{B}\right)\right| \frac{1+e^{i \pi\left|\lambda_{B}\right|}\left[\frac{\delta b_{4}\left(\frac{2 c_{B}}{k}\right)^{2\left|\lambda_{B}\right|}}{16 \pi\left|\lambda_{B}\right| c_{B}}\right]}{1-e^{i \pi\left|\lambda_{B}\right|}\left[\frac{\delta b_{4}\left(\frac{2 c_{B}}{k}\right)^{2\left|\lambda_{B}\right|}}{16 \pi\left|\lambda_{B}\right| c_{B}}\right]},
$$


so that the S-matrix (1.2) reduces to

$$
\begin{aligned}
T_{S}(\sqrt{s}, \theta)= & -16 \pi i c_{B}\left(\cos \left(\pi \lambda_{B}\right)-1\right) \delta(\theta)+8 i c_{B} \sin \left(\pi \lambda_{B}\right) \operatorname{Pv}\left(\cot \left(\frac{\theta}{2}\right)\right) \\
& +8 c_{B}\left|\sin \left(\pi \lambda_{B}\right)\right| \frac{1+e^{i \pi\left|\lambda_{B}\right|} \frac{A_{R}}{k^{2 \mid \lambda_{B}} \mid}}{1-e^{i \pi\left|\lambda_{B}\right|} \frac{A_{R}}{k^{2 \mid \lambda_{B}} \mid}}, \\
A_{R}= & {\left[\frac{\delta b_{4}\left(2 c_{B}\right)^{2\left|\lambda_{B}\right|}}{16 \pi\left|\lambda_{B}\right| c_{B}}\right] . }
\end{aligned}
$$

On the other hand the S-matrix obtained by solving the Schroedinger equation (1.7) subject to the boundary conditions (1.10) has already been determined in [7] and we rederive it in the appendix A.2. ${ }^{7}$ It turns out that

$$
\begin{aligned}
T_{N R}= & -16 \pi i c_{B}\left(\cos \left(\pi \lambda_{B}\right)-1\right) \delta(\theta)+8 i c_{B} \sin \left(\pi \lambda_{B}\right) \operatorname{Pv}\left(\cot \frac{\theta}{2}\right) \\
& +8 c_{B}\left|\sin \pi \lambda_{B}\right| \frac{1+e^{i \pi\left|\lambda_{B}\right|} \frac{A_{N R}}{k^{2\left|\lambda_{B}\right|}}}{1-e^{i \pi\left|\lambda_{B}\right|} \frac{A_{N R}}{k^{2\left|\lambda_{B}\right|}}} \\
A_{N R}= & \frac{-1}{w}\left(\frac{2}{R}\right)^{2\left|\lambda_{B}\right|} \frac{\Gamma\left(1+\left|\lambda_{B}\right|\right)}{\Gamma\left(1-\left|\lambda_{B}\right|\right)} .
\end{aligned}
$$

The $S$-matrices (1.12) and (1.15) are identical in structure. They agree in all details provided we identify

$$
-w\left(c_{B} R\right)^{2\left|\lambda_{B}\right|}=\frac{c_{B}}{\delta b_{4}}\left(16 \pi\left|\lambda_{B}\right| \frac{\Gamma\left(1+\left|\lambda_{B}\right|\right)}{\Gamma\left(1-\left|\lambda_{B}\right|\right)}\right) .
$$

(1.16) determines the hitherto unknown dependence of the boundary condition parameter $w R^{2\left|\lambda_{B}\right|}$ as a function of $\delta b_{4}$.

In summary, in the near threshold limit, the S-matrix (1.2) agrees perfectly with the Smatrix computed from the Schroedinger equation (1.7) subject to the boundary conditions

$$
\psi_{0}(r) \propto\left(r^{\left|\lambda_{B}\right|}-\frac{\frac{c_{B}}{\delta b_{4}}\left(16 \pi\left|\lambda_{B}\right| \frac{\Gamma\left(1+\left|\lambda_{B}\right|\right)}{\Gamma\left(1-\left|\lambda_{B}\right|\right)}\right)}{\left(r c_{B}^{2}\right)^{\left|\lambda_{B}\right|}}\right) .
$$

\footnotetext{
${ }^{7}$ More precisely in the appendix A.2 we show that the Schroedinger equation described above has a scattering solution that takes the form

$$
\begin{aligned}
\psi(\vec{r}) & =e^{i k x}+\zeta(\vec{r}), \\
\zeta(\vec{r}) & =\frac{e^{-\frac{i \pi}{4}} e^{i k r} h(\theta)}{\sqrt{2 \pi k r}}+\mathcal{O}\left(\frac{1}{r^{\frac{3}{2}}}\right) \\
h(\theta) & =2 \pi\left(\cos \left(\pi \lambda_{B}\right)-1\right) \delta(\theta)-\sin \left(\pi \lambda_{B}\right) \operatorname{Pv}\left(\cot \frac{\theta}{2}\right)+i\left|\sin \left(\pi \lambda_{B}\right)\right| \frac{1+e^{i \pi\left|\lambda_{B}\right|}\left[\frac{-1}{w}\left(\frac{2}{k R}\right)^{2\left|\lambda_{B}\right|} \frac{\Gamma\left(1+\left|\lambda_{B}\right|\right)}{\Gamma\left(1-\left|\lambda_{B}\right|\right)}\right]}{1-e^{i \pi\left|\lambda_{B}\right|}\left[\frac{-1}{w}\left(\frac{2}{k R}\right)^{2\left|\lambda_{B}\right|} \frac{\Gamma\left(1+\left|\lambda_{B}\right|\right)}{\Gamma\left(1-\left|\lambda_{B}\right|\right)}\right]}
\end{aligned}
$$

The non-relativistic limit of the usual invariant scattering amplitude is given by

$$
T_{N R}=-8 i c_{B} h(\theta) .
$$


As we have emphasized above, however, the effect of the modified boundary conditions on a process at momentum scale $k$ is measured by $w(R k)^{2\left|\lambda_{B}\right|}$. It follows from (1.16) that in the current situation, the effect of the modified boundary conditions on a process at momentum scale $k$ is measured by

$$
M=\frac{c_{B}}{\delta b_{4}}\left(\frac{k}{c_{B}}\right)^{2\left|\lambda_{B}\right|}\left(16 \pi\left|\lambda_{B}\right| \frac{\Gamma\left(1+\left|\lambda_{B}\right|\right)}{\Gamma\left(1-\left|\lambda_{B}\right|\right)}\right) .
$$

Note that $M$ is held fixed in the near threshold scaling limit (1.5). The modified boundary condition can be ignored when $M \rightarrow 0$. $M$ tends to zero in, for instance, the usual nonrelativistic limit (where $k$ is scaled to zero with all other parameters like $\delta b_{4}$ held fixed). Consequently $w R^{2\left|\lambda_{B}\right|}$ is effectively zero in the quantum mechanical description of the usual non-relativistic limit, explaining why (1.2) reduces to the $w=0$ Aharonov-BohmRuijsenaars [2, 26] S matrix in this limit, as noted in [9].

The agreement of the S-matrix (1.2) (and in particular of its poles) with (1.15) in the near threshold limit immediately demonstrates that the spectrum of near threshold bound states of the singlet particle - antiparticle sector of (1.1) agrees with the spectrum of bound states of the Schroedinger equation (1.7) subject to the boundary conditions (1.17).

The scattering matrices $T_{S}$ and $T_{N R}$ are quite involved functions of $k$ and $\lambda_{B}$; for this reason we view the matching of these two functions in the appropriate limit as a rather nontrivial test of the conjectured $\mathrm{S}$ matrix (1.2). Note that $T_{S}$ would not have matched with $T_{N R}$ without the the additional factor $\frac{\sin \left(\pi \lambda_{B}\right)}{\pi \lambda_{B}}$ invoked in [9]. As a consequence the results of this note provide indirect support to the modified crossing symmetry properties for the S matrix of matter Chern-Simons theories conjectured in [9].

In this paper we have argued that the $\mathrm{S}$ matrix (1.2) may be derived from a Schroedinger equation in a particular scaling limit. Perhaps it is possible to derive the full relativistic formula (1.2) from the solution to an appropriate Schroedinger equation in lightcone slicing; we leave the further investigations of this issue to future work.

\section{Acknowledgments}

We would like to thank K. Inbasekar, S. Jain, J. Maldacena, D. Son, T. Takimi, S. Wadia and S. Yokoyama for useful discussions and S. Jain for comments on the manuscript. S.M. would like to thank the University of Chicago and especially the Institute for Advanced Study for hospitality when this work was initiated. We would also like to acknowledge our debt to the people of India for their steady and generous support to research in the basic sciences.

\section{A The quantum mechanics of anyons with point like interactions}

In the main text we have followed $[7,25]$ to assert that point like interactions between anyons effectively impose modified local boundary conditions on the Schroedinger equation in the relative coordinates. This assertion may appear unfamiliar as contact interactions usually lead to delta function potentials for relative coordinates. In fact these viewpoints 
are equivalent. In the subsections A.1.1 and A.1.2 below we demonstrate that the correct treatment of the two dimensional delta function at $\lambda_{B}=0$ does, in fact, effectively modify the boundary conditions at the origin and has no other effect. Moreover the boundary conditions so obtained agree with $\lambda_{B} \rightarrow 0$ limit of the boundary conditions (1.10).

In subsection A.2 we proceed to rederive the scattering amplitude for the Schroedinger equation (1.7) subject to the boundary conditions (1.10); our results agree with those of [7].

\section{A.1 Quantum mechanics with a two dimensional delta function}

\section{A.1.1 Renormalization of the coupling constant}

In this section we review the dynamics of the quantum mechanical system governed by the two dimensional Schroedinger equation

$$
-\frac{\nabla^{2}}{2 m} \psi(\vec{x})+V(\vec{x}) \psi(\vec{x})=\frac{k^{2}}{2 m} \psi(\vec{x})
$$

where $V(\vec{x})$ is taken to be proportional to a suitably renormalized version of the attractive two dimensional $\delta$ function. This system has been studied in great detail in several papers (see e.g. [27]); we review the principal results.

Let

$$
\psi(\vec{x})=\int \frac{d^{2} k}{(2 \pi)^{2}} e^{i \vec{k} \cdot \vec{x}} \widetilde{\psi}(\vec{k}) .
$$

The time independent solution of (A.1) that describes the scattering of an incoming particle with momentum $\vec{k}$ off an arbitrary potential $V(x)$ is given by the solution to the LippmannSchwinger equation

$$
\widetilde{\psi}(\vec{p})=(2 \pi)^{2} \delta^{2}(\vec{p}-\vec{k})+2 m \int \frac{d^{2} q}{(2 \pi)^{2}} \frac{\widetilde{V}(\vec{q}) \widetilde{\psi}(\vec{p}-\vec{q})}{k^{2}-p^{2}+i \epsilon} .
$$

Let $V(x)=-g \delta^{2}(\vec{x})$ so that its Fourier transform is given by $\widetilde{V}(\vec{k})=-g$. Plugging into (A.3) we find

$$
\widetilde{\psi}(\vec{p})=(2 \pi)^{2} \delta^{2}(\vec{p}-\vec{k})-\frac{2 m g A(\vec{k})}{k^{2}-p^{2}+i \epsilon},
$$

where

$$
A(\vec{k})=\frac{1}{1-2 m g \int \frac{d^{2} p}{(2 \pi)^{2}} \frac{1}{p^{2}-k^{2}-i \epsilon}} .
$$

The integral in (A.5) diverges logarithmically. Evaluating the integral with a cut off $\Lambda$ we have

$$
A(\vec{k})=\frac{1}{1-\frac{m g}{2 \pi} \ln \left(\frac{\Lambda^{2}}{-k^{2}}\right)} .
$$

The function $A(\vec{k})$ is proportional to the scattering amplitude of our quantum mechanical system. In order to define a sensible scattering problem we must regulate and renormalize (A.6) by choosing the coupling constant $g$ to scale to zero logarithmically with the cut off $\Lambda$. We choose $g(\Lambda)$ so that

$$
\frac{1}{g(\Lambda)}=\frac{1}{g_{R}(\mu)}+\frac{m}{2 \pi} \ln \left(\frac{\Lambda^{2}}{\mu^{2}}\right)
$$


where the renormalized coupling $g_{R}(\mu)$ is held fixed as $\Lambda$ is taken to infinity. $g_{R}(\mu)$ is, of course, a function of the renormalization scale $\mu$. (A.6) now takes the form ${ }^{8}$

$$
A(\vec{k})=\frac{1}{1-\frac{m g_{R}}{2 \pi} \ln \left(\frac{\mu^{2}}{-k^{2}}\right)} .
$$

\section{A.1.2 Description in terms of modified boundary conditions}

We will now find an alternative effective description of the renormalized two dimensional delta function in terms of modified boundary conditions at $r=0$. For this purpose it will prove convenient to work in position rather than momentum space. For this reason we regulate the $\delta$ function potential as the 'circular square well'

$$
\begin{aligned}
V(r) & =-\frac{g}{\pi r_{0}^{2}} ; & & r<r_{0}, \\
& =0 ; & & r>r_{0} .
\end{aligned}
$$

Let us now study rotationally invariant solutions of the two dimensional Schroedinger equation with the potential (A.11). ${ }^{9}$ Clearly the most general regular (at $r=0$ ) solution to the Schroedinger equation takes the form

$$
\begin{aligned}
a J_{0}(l r) & ; \quad r<r_{0}, \\
c J_{0}(k r)+d Y_{0}(k r) ; & r>r_{0},
\end{aligned}
$$

where,

$$
l^{2}=2 m\left(\frac{g}{\pi r_{0}^{2}}+E\right), \quad k^{2}=2 m E .
$$

The requirement of continuity of the wave function and its first derivative across $r=r_{0}$ determines $d$ and $c$ in terms of $a$. In the small $r_{0}$ limit it is easily verified that

$$
\frac{d}{c}=\frac{-1}{\frac{2}{m g}+\frac{2}{\pi}\left[\gamma+\ln \left(\frac{k r_{0}}{2}\right)\right]},
$$

where, $\gamma$ is Euler-Mascheroni constant.

As in the previous section (A.14) does not have a well defined $r_{0} \rightarrow 0$ limit. In order that the l.h.s. of (A.14) is well defined as $r_{0} \rightarrow 0$ we must choose $g$ to be a function of $r_{0}$ and take $g$ to zero as $r_{0}$ is scaled to zero, keeping $g_{R}$ fixed where

$$
\frac{1}{g_{R}(\mu)}=\frac{1}{g\left(r_{0}\right)}+\frac{m}{\pi}\left[\ln \left(\frac{r_{0} \mu}{2}\right)+\gamma\right] \text {. }
$$

${ }^{8}$ As an application notice that the scattering amplitude (A.10) has a pole at

$$
k^{2}=-\mu^{2} e^{-\frac{2 \pi}{m g_{R}}}
$$

implying that our renormalized $\delta$ function potential quantum mechanics has a single bound state with binding energy

$$
E=-\frac{\mu^{2}}{2 m} e^{-\frac{2 \pi}{m g_{R}}}
$$

\footnotetext{
${ }^{9}$ Only rotationally invariant solutions are affected by the potential (A.11) in the limit $r_{0} \rightarrow 0$, as the wave function at nonzero angular momentum dies rapidly at small $r$ due to the angular momentum barrier.
} 
Note that (A.15) agrees exactly with (A.7) under the replacement $\frac{\mu r_{0} e^{\gamma}}{2} \rightarrow \frac{\mu}{\Lambda}$.

Implementing this limit we find

$$
\frac{d}{c}=\frac{-1}{\frac{2}{m g_{R}}+\frac{2}{\pi} \ln \left(\frac{k}{\mu}\right)} .
$$

It follows that the Schroedinger problem with a delta function potential with renormalized strength $g_{R}$ is equivalent to the free Schroedinger equation subject to the $r \rightarrow 0$ boundary condition

$$
\psi_{0}(r) \propto\left[\left(-\frac{2}{m g_{R}}-\frac{2}{\pi} \ln \frac{k}{\mu}\right) J_{0}(k r)+Y_{0}(k r)\right] .
$$

Using the small argument expansions

$$
J_{0}(k r)=1+\mathcal{O}\left((k r)^{2}\right), \quad Y_{0}(k r)=\frac{2}{\pi} \ln \left(\frac{k r}{2}\right)+2 \frac{\gamma}{\pi}+\mathcal{O}\left((k r)^{2} \ln (k r)\right),
$$

we see that the $k$ dependence cancels from (A.17) and the boundary condition on $\psi(r)$ takes the local form

$$
\psi_{0}(r) \propto\left[\left(-\frac{2}{m g_{R}}+2 \frac{\gamma}{\pi}\right)+\frac{2}{\pi} \ln \left(\frac{\mu r}{2}\right)+\mathcal{O}\left(r^{2} \ln r\right)\right] .
$$

In summary, the Schroedinger equation in the presence of a renormalized $\delta$ function potential is exactly equivalent to the free Schroedinger equation subject to the local boundary conditions (A.19) at the origin.

It is easily verified that the boundary conditions (A.19) are obtained as a limit of the Amelino-Camelia-Bak boundary conditions (1.10) if we set

$$
w=-1+\left|\lambda_{B}\right|\left(-\frac{2 \pi}{m g_{R}}+2 \gamma+2 \ln \left(\frac{\mu R}{2}\right)\right)
$$

and take the limit $\left|\lambda_{B}\right| \rightarrow 0$. In other words the usual (i.e. $\delta$ function) description of contact interactions is indeed equivalent to the appropriate $|\nu| \rightarrow 0$ limit of the Schroedinger equation (1.7) subject to the boundary conditions (1.10). This suggests that the boundary conditions (1.10) do indeed capture the effect of contact interactions at general $\lambda_{B}$. This has been argued to be true in $[7,25]$.

\section{A.2 Derivation of the scattering amplitude}

In this section we will derive the scattering amplitude for the Schroedinger equation (1.7) subject to the boundary conditions (1.10). We assume $|\nu|<1$.

We wish to find scattering state solutions at energy $E=\frac{k^{2}}{2 m}$ of the Schroedinger equation for this particle; i.e. $k$ is the magnitude of the momentum of the particle incident on the solenoid. The most general solution of the Schroedinger equation that meets the boundary conditions for $\psi_{m}(r)$ at the origin $(m \neq 0)$ is

$$
\psi(\vec{r})=\sum_{n>0} a_{n} e^{i n \theta} J_{n+\nu}(k r)+\sum_{n>0} a_{-n} e^{-i n \theta} J_{n-\nu}(k r)+a_{0} J_{|\nu|}(k r)+b_{0} J_{-|\nu|}(k r) .
$$


The scattering solution we wish to find obeys the boundary condition (1.10); moreover at large $r$ its ingoing piece (part proportional to $e^{-i k r}$ ) must reduce to that of the incoming wave $e^{i k x}$. It is not difficult to see that the unique solution that meets our boundary conditions is given by (see appendix $\mathrm{C}$ of [9] for the detailed derivation for the special case $w=0$ )

$$
\begin{aligned}
\psi(\vec{r})= & \sum_{n=1}^{\infty} i^{n} e^{-i \frac{\pi \nu}{2}} J_{n+\nu}(k r) e^{i n \theta}+\sum_{n=1}^{\infty} i^{n} e^{i \frac{\pi \nu}{2}} J_{n-\nu}(k r) e^{-i n \theta} \\
& +\frac{\Gamma(|\nu|+1)\left(\frac{2}{k}\right)^{|\nu|} J_{|\nu|}(k r)+w R^{2|\nu|} \Gamma(1-|\nu|)\left(\frac{k}{2}\right)^{|\nu|} J_{-|\nu|}(k r)}{\Gamma(|\nu|+1)\left(\frac{2}{k}\right)^{|\nu|} e^{i \frac{\pi|\nu|}{2}}+w R^{2|\nu|} \Gamma(1-|\nu|)\left(\frac{k}{2}\right)^{|\nu|} e^{-i \frac{\pi|\nu|}{2}}} .
\end{aligned}
$$

At large $r, \psi(\vec{r})$ reduces to

$$
\frac{1}{\sqrt{2 \pi k r}}\left(e^{i \frac{\pi}{4}} \delta(\theta-\pi) e^{-i k r}+H(\theta) e^{-i \frac{\pi}{4}} e^{i k r}\right)
$$

where,

$$
\begin{aligned}
H(\theta)= & \sum_{n=1}^{\infty}\left(e^{-i \pi \nu} e^{i n \theta}+e^{i \pi \nu} e^{-i n \theta}\right) \\
& +\frac{\Gamma(|\nu|+1)\left(\frac{2}{k}\right)^{|\nu|} e^{-i \frac{\pi|\nu|}{2}}+w R^{2|\nu|} \Gamma(1-|\nu|)\left(\frac{k}{2}\right)^{|\nu|} e^{i \frac{\pi|\nu|}{2}}}{\Gamma(|\nu|+1)\left(\frac{2}{k}\right)^{|\nu|} e^{i \frac{\pi|\nu|}{2}}+w R^{2|\nu|} \Gamma(1-|\nu|)\left(\frac{k}{2}\right)^{|\nu|} e^{-i \frac{\pi|\nu|}{2}}} .
\end{aligned}
$$

Now we can write

$$
\begin{aligned}
& \sum_{n=1}^{\infty}\left(e^{-i \pi \nu} e^{i n \theta}+e^{i \pi \nu} e^{-i n \theta}\right)=\left(\sum_{n=1}^{\infty} 2 \cos (\pi \nu) \cos (n \theta)\right)+\left(\sum_{n=1}^{\infty} 2 \sin (\pi \nu) \sin (n \theta)\right) \\
& =\left(\cos (\pi \nu)+\sum_{n=1}^{\infty} 2 \cos (\pi \nu) \cos (n \theta)\right)-\cos (\pi \nu)+\left(\sum_{n=1}^{\infty} 2 \sin (\pi \nu) \sin (n \theta)\right) \\
& =2 \pi \cos (\pi \nu) \delta(\theta)-\cos (\pi \nu)+\left(\sum_{n=1}^{\infty} 2 \sin (\pi \nu) \sin (n \theta)\right) \\
& =2 \pi \cos (\pi \nu) \delta(\theta)+\sin (\pi \nu) \operatorname{Pv}\left(\cot \left(\frac{\theta}{2}\right)\right)-\cos (\pi \nu) .
\end{aligned}
$$

Substituting in (A.22)

$$
\begin{aligned}
H(\theta)= & 2 \pi \cos (\pi \nu) \delta(\theta)+\sin (\pi \nu) \operatorname{Pv}\left(\cot \left(\frac{\theta}{2}\right)\right) \\
& +\frac{\Gamma(|\nu|+1)\left(\frac{2}{k}\right)^{|\nu|} e^{-i \frac{\pi|\nu|}{2}}+w R^{2|\nu|} \Gamma(1-|\nu|)\left(\frac{k}{2}\right)^{|\nu|} e^{i \frac{\pi|\nu|}{2}}}{\Gamma(|\nu|+1)\left(\frac{2}{k}\right)^{|\nu|} e^{i \frac{\pi|\nu|}{2}}+w R^{2|\nu|} \Gamma(1-|\nu|)\left(\frac{k}{2}\right)^{|\nu|} e^{-i \frac{\pi|\nu|}{2}}}-\cos (\pi \nu) \\
= & 2 \pi \cos (\pi \nu) \delta(\theta)+\sin (\pi \nu) \operatorname{Pv}\left(\cot \left(\frac{\theta}{2}\right)\right) \\
& -i \sin (\pi|\nu|) \frac{\Gamma(|\nu|+1)\left(\frac{2}{k}\right)^{|\nu|} e^{i \pi|\nu|}-w R^{2|\nu|} \Gamma(1-|\nu|)\left(\frac{k}{2}\right)^{|\nu|}}{\Gamma(|\nu|+1)\left(\frac{2}{k}\right)^{|\nu|} e^{i \pi|\nu|}+w R^{2|\nu|} \Gamma(1-|\nu|)\left(\frac{k}{2}\right)^{|\nu|}}
\end{aligned}
$$


In order to compute the scattering amplitude, we must rewrite the wave function as a plane wave plus a scattered piece; at large $r$

$$
\psi(r)=e^{i k x}+\frac{h(\theta) e^{-i \frac{\pi}{4}} e^{i k r}}{\sqrt{2 \pi k r}} .
$$

We find

$$
h(\theta)=H(\theta)-2 \pi \delta(\theta),
$$

so that

$$
\begin{aligned}
h(\theta)= & 2 \pi(\cos (\pi \nu)-1) \delta(\theta)+\sin (\pi \nu) \operatorname{Pv}\left(\cot \left(\frac{\theta}{2}\right)\right) \\
& -i \sin (\pi|\nu|) \frac{\Gamma(|\nu|+1)\left(\frac{2}{k}\right)^{|\nu|} e^{i \pi|\nu|}-w R^{2|\nu|} \Gamma(1-|\nu|)\left(\frac{k}{2}\right)^{|\nu|}}{\Gamma(|\nu|+1)\left(\frac{2}{k}\right)^{|\nu|} e^{i \pi|\nu|}+w R^{2|\nu|} \Gamma(1-|\nu|)\left(\frac{k}{2}\right)^{|\nu|}} .
\end{aligned}
$$

This yields (1.13).

Open Access. This article is distributed under the terms of the Creative Commons Attribution License (CC-BY 4.0), which permits any use, distribution and reproduction in any medium, provided the original author(s) and source are credited.

\section{References}

[1] E. Witten, Quantum field theory and the Jones polynomial, Commun. Math. Phys. 121 (1989) 351 [inSPIRE].

[2] Y. Aharonov and D. Bohm, Significance of electromagnetic potentials in the quantum theory, Phys. Rev. 115 (1959) 485 [InSPIRE].

[3] F. Wilczek, Fractional statistics and anyon superconductivity, World Scientific, Singapore (1990) [INSPIRE].

[4] R. Jackiw, Dynamical symmetry of the magnetic vortex, Annals Phys. 201 (1990) 83 [INSPIRE].

[5] D. Bak, R. Jackiw and S.-Y. Pi, Non-Abelian Chern-Simons particles and their quantization, Phys. Rev. D 49 (1994) 6778 [hep-th/9402057] [INSPIRE].

[6] D. Bak and O. Bergman, Perturbative analysis of non-Abelian Aharonov-Bohm scattering, Phys. Rev. D 51 (1995) 1994 [hep-th/9403104] [INSPIRE].

[7] G. Amelino-Camelia and D. Bak, Schrödinger selfadjoint extension and quantum field theory, Phys. Lett. B 343 (1995) 231 [hep-th/9406213] [INSPIRE].

[8] S. Park and D. Bak, Exact 4-point scattering amplitude of the superconformal Schrödinger Chern-Simons theory, J. Korean Phys. Soc. 60 (2012) 714 [arXiv:1203.0068] [INSPIRE].

[9] S. Jain et al., Unitarity, crossing symmetry and duality of the $S$-matrix in large- $N$ Chern-Simons theories with fundamental matter, arXiv:1404.6373 [INSPIRE].

[10] O. Aharony, G. Gur-Ari and R. Yacoby, $D=3$ bosonic vector models coupled to Chern-Simons gauge theories, JHEP 03 (2012) 037 [arXiv:1110.4382] [INSPIRE]. 
[11] S. Giombi et al., Chern-Simons theory with vector fermion matter, Eur. Phys. J. C 72 (2012) 2112 [arXiv:1110.4386] [INSPIRE].

[12] J. Maldacena and A. Zhiboedov, Constraining conformal field theories with a higher spin symmetry, J. Phys. A 46 (2013) 214011 [arXiv:1112.1016] [INSPIRE].

[13] J. Maldacena and A. Zhiboedov, Constraining conformal field theories with a slightly broken higher spin symmetry, Class. Quant. Grav. 30 (2013) 104003 [arXiv:1204.3882] [InSPIRE].

[14] C.-M. Chang, S. Minwalla, T. Sharma and X. Yin, ABJ triality: from higher spin fields to strings, J. Phys. A 46 (2013) 214009 [arXiv:1207.4485] [InSPIRE].

[15] O. Aharony, G. Gur-Ari and R. Yacoby, Correlation functions of large-N Chern-Simons-matter theories and bosonization in three dimensions, JHEP 12 (2012) 028 [arXiv: 1207.4593] [INSPIRE].

[16] S. Jain, S.P. Trivedi, S.R. Wadia and S. Yokoyama, Supersymmetric Chern-Simons theories with vector matter, JHEP 10 (2012) 194 [arXiv:1207.4750] [INSPIRE].

[17] S. Yokoyama, Chern-Simons-Fermion vector model with chemical potential, JHEP 01 (2013) 052 [arXiv: 1210.4109] [INSPIRE].

[18] G. Gur-Ari and R. Yacoby, Correlators of large- $N$ fermionic Chern-Simons vector models, JHEP 02 (2013) 150 [arXiv:1211.1866] [InSPIRE].

[19] O. Aharony, S. Giombi, G. Gur-Ari, J. Maldacena and R. Yacoby, The thermal free energy in large-N Chern-Simons-matter theories, JHEP 03 (2013) 121 [arXiv:1211.4843] [INSPIRE].

[20] S. Jain et al., Phases of large- $N$ vector Chern-Simons theories on $S^{2} \times S^{1}$, JHEP 09 (2013) 009 [arXiv:1301.6169] [INSPIRE].

[21] T. Takimi, Duality and higher temperature phases of large- $N$ Chern-Simons matter theories on $S^{2} \times S^{1}$, JHEP 07 (2013) 177 [arXiv: 1304.3725] [INSPIRE].

[22] S. Jain, S. Minwalla and S. Yokoyama, Chern-Simons duality with a fundamental boson and fermion, JHEP 11 (2013) 037 [arXiv:1305.7235] [INSPIRE].

[23] Y. Frishman and J. Sonnenschein, Breaking conformal invariance - large-N Chern-Simons theory coupled to massive fundamental fermions, JHEP 12 (2013) 091 [arXiv:1306.6465] [INSPIRE].

[24] S. Yokoyama, A note on large- $N$ thermal free energy in supersymmetric Chern-Simons vector models, JHEP 01 (2014) 148 [arXiv:1310.0902] [INSPIRE].

[25] S.-J. Kim and C.-K. Lee, Quantum description of anyons: role of contact terms, Phys. Rev. D 55 (1997) 2227 [hep-th/9606054] [inSPIRE].

[26] S.N.M. Ruijsenaars, The Aharonov-Bohm effect and scattering theory, Annals Phys. 146 (1983) 1 [INSPIRE].

[27] R. Jackiw, Delta function potentials in two-dimensional and three-dimensional quantum mechanics, (1991) [INSPIRE]. 